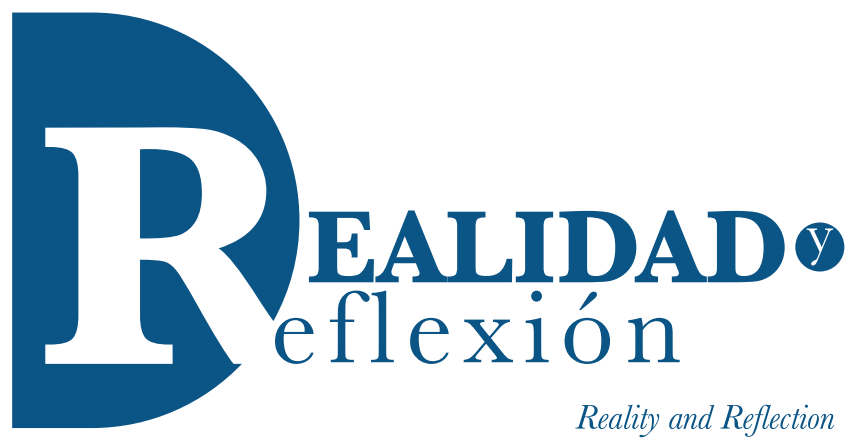

Año 19, Nº 50 San Salvador, El Salvador, Centroamérica. Revista Semestral julio-diciembre 2019

YEAR 19, No 50 SAN SALVADOR, EL SALVADOR, CENTRAL AMERICA. SEMESTRAL JOURNAL JLUIO- DICIEMBRE 2019

\title{
Análisis de redes sociales: Identificación de comunidades virtuales en Twitter
}

\section{Social Network Analysis: Identifying Virtual Communities on Twitter}

\section{RESUMEN}

James Edward Humberstone Morales Ingeniero en Ciencias de la Computación y Maestro en Informática Aplicada a Redes por la Universidad Francisco Gavidia Docente de la Facultad de Ingeniería y Sistemas Investigador del Laboratorio de Nanotecnología y Centro de Modelaje Matemático en la Universidad Francisco Gavidia jhumberstone@ufg.edu.sv

Fernando José Alvarez Alens Estudiante de Ingeniería en Telecomunicaciones de la Universidad Francisco Gavidia ia.feralens@ufg.edu.sv Recibido: 27 de febrero de 2019 Aprobado: 29 de noviembre de 2019 DOI: $10.5377 /$ ryr.v50i50.9095

El Análisis de redes sociales consiste en examinar estructuras de entidades: datos, objetos, grupos de personas, etc.; y sus interacciones dentro de una comunidad centrándose en las relaciones que existen entre ellas. En la presente investigación se expone una metodología para determinar las comunidades virtuales que interactúan en la plataforma de la red social Twitter. Como validación, se realizó la captura de datos de tres eventos mediáticos relacionados a la acción política de los candidatos a la presidencia de El Salvador 2019.

Palabras clave: análisis de redes sociales; minería de datos; análisis de tweets; comunidad virtual.

\section{ABSTRACT}

The social network analysis consists of examining structures of entities: data, objects, groups of people, etc.; and their interactions within a community centered on the relationships that exist between them. In the present investigation a methodology is exposed to determine the virtual communities that interact in the platform of the social network Twitter. As validation, the data capture of three media events related to the political action of the candidates for the presidency of El Salvador 2019 was carried out.

Key words: Social network analysis, Data mining, Tweet analysis, Online Community. 


\section{Introducción}

El Análisis de las Redes Sociales (ARS) es fruto del aporte de la matemática (análisis cuantitativo por medio de la teoría de grafos), informática (software de análisis de redes) y las ciencias humanísticas (análisis cualitativo por medio de sociogramas); y consiste en estudiar la relación entre actores: datos, objetos, grupos de personas, organizaciones, etc.

Tal como indica el Knowledge Center de IBM, el ARS consiste en examinar estructuras de grupo y flujos de comunicación dentro de un gráfico de red centrándose en las relaciones que existen entre entidades.

Según Dettemer y Reyna (2014), las aplicaciones de las ARS han crecido en la última década, se han hecho estudios sobre comunidades virtuales, redes de conocimiento, redes y sistemas de innovación, redes y estructuras criminales, redes de acción política, estudios sobre flujos de transporte y redes de ciudades, entre otros.

A partir del año 1995 surgieron las redes sociales en Internet, sitios web dedicados a brindar servicios de comunicación. En la década del año 2000 se popularizaron con el surgimiento de Facebook y Twitter, según Dannbraun (2017). Hoy en día prácticamente todo el mundo tiene un perfil en las redes sociales, diariamente se genera y comparte una inmensidad de contenido que puede ser analizado para descubrir información relevante respecto a las comunidades virtuales. Esta información puede usarse para conocer sus intereses, establecer redes de acción política y social, así como fácilmente una empresa puede obtener el grado de aceptación de sus clientes hacia un producto y/o servicio. Dicho esto, surge la interrogante ¿Qué herramientas podemos utilizar para realizar ARS y determinar comunidades virtuales en los servicios de internet como Twitter? ¿Cuál es el procedimiento para recopilar, analizar y visualizar la información?

Para dar respuesta a estas interrogantes se aprovechó la coyuntura de las elecciones presidenciales 2019 de El Salvador. Se recopiló los tweets generados por los actores políticos en contienda por medio de la Stream Api de Twitter y posteriormente se creó un procedimiento para realizar el análisis y visualización utilizando la herramienta Gephi, programa Open Source para explorar y manipular redes según Bastian, Heymann y Jacomy (2009).

\section{Método}

Este documento tiene como objetivo establecer una metodología para analizar las comunidades virtuales en la red social de Twitter, para proveer a los investigadores salvadoreños, interesados en el ARS, un procedimiento para capturar, analizar $\mathrm{y}$ visualizar los datos.

Según Twitter (2019), su Stream Api permite hacer capturas en tiempo real, por tanto, es ideal para monitorear la reacción e interacción de los usuarios de Twitter en el desarrollo de un evento tal como la acción política de los candidatos.

En ciencias humanísticas las interacciones de las comunidades se analizan por medio de sociogramas. Para un análisis cuantitativo, los sociogramas se apoyan en la teoría de grafos. De manera que en el grafo cada actor (usuario, hashtag, tweet) es representado por un nodo y 
los lazos (mensajes directos, menciones, retweet) son representados por aristas.

Para el análisis y visualización de grafos utilizamos la herramienta de Gephi versión 0.9.2 con el plugin Twitter Streaming Importer ${ }^{1}$ tal como lo indica Totet (2016).

\section{Preparación del entorno para captura de datos}

Dentro de Gephi hay que instalar el plugin Twitter Streaming Importer (Consultar guía de instalación en anexo). Para poder acceder a la red datos de Twitter es necesario ingresar las credenciales de desarrollador tal como lo menciona Humberstone (2018). Una vez ingresadas las credenciales, se configura los parámetros de monitoreo, entre ellos: lista de usuarios, locación, palabras claves y tipo de red; finalmente se procede a la recolección de datos.

\section{Recolección de datos}

Una vez recolectados los datos nos dirigimos a la

1 Sitio web para descargar el plugin https://gephi.org/plugins/\#/ plugin/twitter-streaming-importer pestaña "Laboratorio de Datos" para explorar el contenido. Gephi nos provee la información en dos dataset ${ }^{2}$ : uno para los nodos y otro para las aristas. Es importante tener pleno conocimiento del tipo de información obtenida.A continuación, resumimos los campos más relevantes para la investigación. (Ver Tabla 1 y Tabla 2)

\section{Análisis y procesamiento de grafos en Gephi}

Para iniciar el procesamiento del grafo inicial se aplicó las siguientes medidas estadísticas de centralidad que permitirán descubrir información relevante. La medida Grado Medio permite conocer el grado de entrada y salida de un nodo, cuantas veces está conectado un nodo dentro de toda la red. El grado de entrada y el grado de salida definen el rol de influyente y el rol de comunicador, respectivamente dentro de la red.

La medida "Diámetro de red" brinda el número de nodos necesarios para transportarse en toda la red de un extremo a otro. Además, esta medida proporciona tres medidas adicionales muy importantes para el análisis.

2 Conjunto de datos tabulados.

\section{Tabla 1}

Diccionario de datos del dataset nodos

\begin{tabular}{|c|c|c|}
\hline Campo & Descripción & Uso \\
\hline created_at & Fecha de creación del perfil de usuario & Conocer si son usuarios creados recientemente. \\
\hline timeset & $\begin{array}{l}\text { Fecha y hora en que se recuperaron los datos. } \\
\text { Muestra la frecuencia con la que se realiza una } \\
\text { determinada acción }\end{array}$ & $\begin{array}{l}\text { Llevar un registro de las recuperaciones de información, } \\
\text { conociendo la fecha y hora de cada recuperación. }\end{array}$ \\
\hline description & Descripción que cada usuario ha colocado en su perfil & \\
\hline location & $\begin{array}{l}\text { Nombre de la ubicación de donde se realizó la } \\
\text { acción }\end{array}$ & $\begin{array}{l}\text { Conocer datos geográficos de dónde se realizan la } \\
\text { mayor cantidad de acciones }\end{array}$ \\
\hline
\end{tabular}

Nota: El dataset contiene más campos, pero solamente se han listado los campos relevantes para la investigación 
Tabla 2

Diccionario de datos del dataset arista

\begin{tabular}{|c|c|c|}
\hline Campo & Descripción & Uso \\
\hline Kind & $\begin{array}{l}\text { Tipo de acción que se realizó en } \\
\text { Twitter: } \\
\text { - Tweet } \\
\text { - Retweet } \\
\text { - Mention } \\
\text { - Hashtag } \\
\text { - Quote (Mensaje privado o directo) } \\
\text { - Link } \\
\text { - Media }\end{array}$ & $\begin{array}{l}\text { Conocer el tipo de interacción que realizó un usuario hacia otro. } \\
\text { Además de clasificar las interacciones. }\end{array}$ \\
\hline Source & $\begin{array}{l}\text { Nodo fuente, de quién parte una } \\
\text { determinada interacción. }\end{array}$ & \multirow[t]{2}{*}{$\begin{array}{l}\text { Conocer el grado de influencia que tiene un nodo, así como el rol que } \\
\text { desempeña dentro de la red. }\end{array}$} \\
\hline Target & $\begin{array}{l}\text { Nodo destino, hacia quién va dirigida } \\
\text { una determinada interacción. }\end{array}$ & \\
\hline
\end{tabular}

Nota: El dataset contiene más campos, pero solamente se han listado los campos relevantes para la investigación.

- Betweenness (Intermediación): Es una medida que brinda el número de rutas más cortas entre dos nodos que pasan a través de un nodo en particular. Una alta intermediación puede sugerir que el nodo está conectando varias partes de la red, es decir, estos nodos que mantienen la red unida.

- Closeness (Cercanía): Es una medida que indica qué tan cerca está un nodo de todos los demás nodos de una red. Una alta cercanía significa que hay una gran distancia promedio a otros nodos en la red.

- Eccentricity (Excentricidad): Excentricidad es una medida que brinda la distancia entre un nodo y el nodo que está más alejado de él. Una excentricidad alta significa que el nodo más alejado de la red está muy lejos, y una excentricidad baja significa que el nodo más alejado en realidad está bastante cerca.
Después de aplicar las medidas estadísticas se debe aplicar filtros para reducir el número de nodos y aristas dentro del grafo total, de esta manera se obtienen grafos simplificados.

\section{Los filtros utilizados son:}

- Igualdad: Permite filtrar la red según un atributo del nodo, por ejemplo, por tweet; por usuario o por hashtag.

- Topología,opción Rangode grado: Filtrala red según los límites de grado que se especifiquen, por tanto, muestra los nodos que cumplen con el rango de relaciones que se indique.

- Topología, opción Ego: Permite conocer la comunidad de un nodo identificado por su atributo principal.

Finalmente, se debe aplicar algoritmos de distribución para tener una visualización más 
clara de la red y apreciar los roles que juegan los diferentes nodos. Usualmente los algoritmos de distribución siguen un principio básico basado en la fuerza, los nodos conectados se atraen entre sí y los nodos no enlazados se separan.

- Fruchterman Reingold ${ }^{3}$ : Es un tipo de distribución que ordena todos los nodos creando una circunferencia, es funcional para tener una visualización de cada uno de los nodos que conforma la red. (ver figura 9).

- Noverlap: Permite que no exista superposición entre los nodos. Puede darse el caso que nodos de un tamaño grande oculten a nodos pequeños.

- Expansión: Esta distribución permite, como su nombre lo indica, expandir la red sin cambiar el diseño que ya posee.

- Ajuste de etiquetas: Cada nodo representa una información, por lo cual cuenta con una etiqueta que puede ser un tweet, un usuario, un hashtag. Este algoritmo permite que no exista traslape entre etiquetas y pueda ser clara la lectura del contenido del nodo.

\section{Consideraciones para el análisis}

Es importante tener ciertas consideraciones al momento de utilizar la herramienta Gephi para la visualización de la red.

1. Hacer una copia del archivo original. Gephi no cuenta con un botón de deshacer, una vez

3 Fruchterman, T. M. J., \& Reingold, E. M. (1991). Graph Drawing by Force-Directed Placement. Software: Practice and Experience, 21(11). modificado los dataset o la vista del grafo, no es posible regresarlo a sus condiciones iniciales.

2. Una vez encontrada una visualización adecuada de la red utilizar la opción de exportar en un nuevo espacio de trabajo para guardarlo fácilmente.

3. Es recomendable exportar un archivo de grafo en formato gexf, de igual manera, es necesario especificar al momento de guardarlo que se quiere guardar solo el grafo visible, en caso contrario, aunque se haya filtrado se guardará completo.

\section{Resultados}

Para probar la metodología se aprovechó la coyuntura de las elecciones presidenciales del año 2019 y los eventos mediáticos asociados, capturando información de los siguientes experimentos.

\section{Experimento \#1:}

\section{Carmen Aida Lazo y Nayib Bukele}

Este evento tuvo lugar el 19 de enero de 2019, donde el candidato a la presidencia por el partido GANA, Nayib Bukele, y la candidata a la vicepresidencia por el partido ARENA, Carmen Aida Lazo, entraron en un debate de opiniones a través de sus cuentas de Twitter. Este evento desató una oleada de Tweets que duró hasta el siguiente día. Para este evento se determinaron cuáles usuarios desempeñan el rol de intermediario (ver figura 1), influyente (ver figura 2) y comunicador en la red (ver figura 3). 
Tabla 3

Parámetros de búsqueda de experimento \#1

\begin{tabular}{l|l}
\hline Tipo de búsqueda & Lista de usuarios \\
\hline Términos de búsqueda & $\begin{array}{l}\boldsymbol{\bullet} @ \text { carmenaidalazo } \\
\bullet\end{array}$ @nayibbukele \\
\hline Tipo de red & User Network \\
\hline Tiempo de captura & 2 horas \\
\hline
\end{tabular}

\section{@carmenaidalazo}

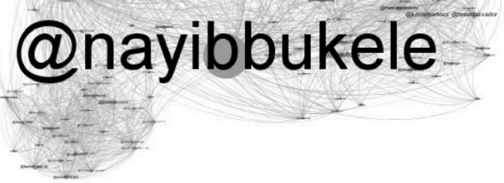

Como resultado obtuvimos un grafo dirigido con 2,093 nodos, usuarios, y 9,726 aristas, interacciones entre los usuarios: retweet, mensaje directo, respuesta o mención.

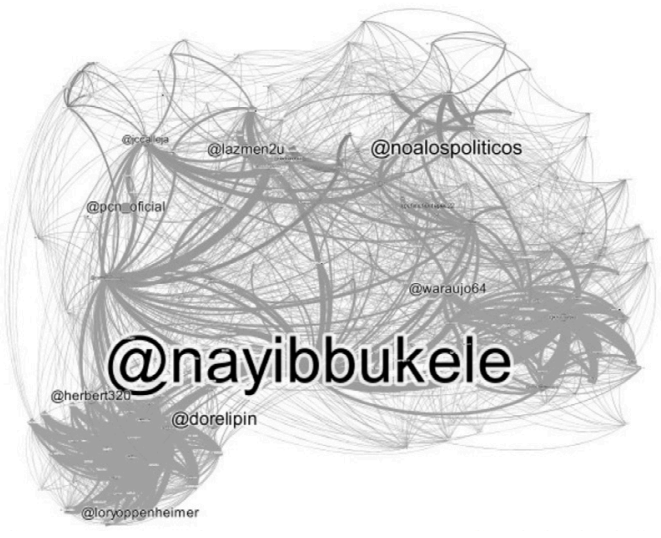

Figura 1. Grafo intermediario. Este grafo indica cuáles usuarios son los intermediarios. Estos usuarios son primordiales para mantener varias secciones de la red unidas, ya que se vuelven el medio o canal para que la información fluya en la red. Fuente: elaboración propia.

Figura 2. Grafo influyente. Indica cuál usuario tiene mayor influencia dentro de la red. Los usuarios destacados tienen mayor presencia dentro de la red; es decir, el usuario con mayor cantidad de retweet, mención y/o respuesta. Fuente: elaboración propia.

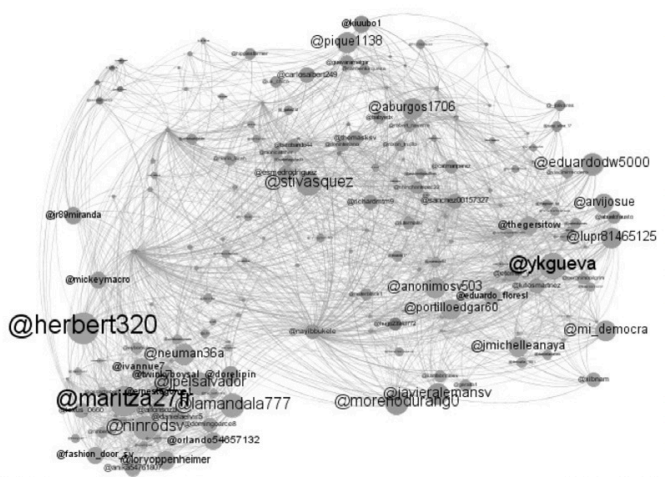

Figura 3. Grafo comunicador. El grafo indica cuales usuarios cumplen el rol de comunicadores. Estos son los usuarios que generan más contenido (tweets, retweets, menciones) dentro de la red. Fuente: elaboración propia. 


\section{Experimento \#2: Revista Factum}

La revista digital Factum publicó el artículo referido al candidato a la presidencia Nayib Bukele denominado "El dinero alcanza cuando los contratos son para amigos de Nayib” (http:// revistafactum.com/contratos-campana-bukele/). Para este evento se generó un grafo que denota las comunidades dentro de la red.

\section{Tabla 4}

Parámetros de búsqueda de experimento \#2

\begin{tabular}{|c|c|}
\hline Tipo de búsqueda & Lista de usuarios \\
\hline Términos de búsqueda & $\begin{array}{l}\text {-@jccalleja } \\
\text {-@ @ayibbukele } \\
\text {-@revistafactum }\end{array}$ \\
\hline Tipo de red & Full Twitter Network \\
\hline Tiempo de captura & 1 hora \\
\hline
\end{tabular}

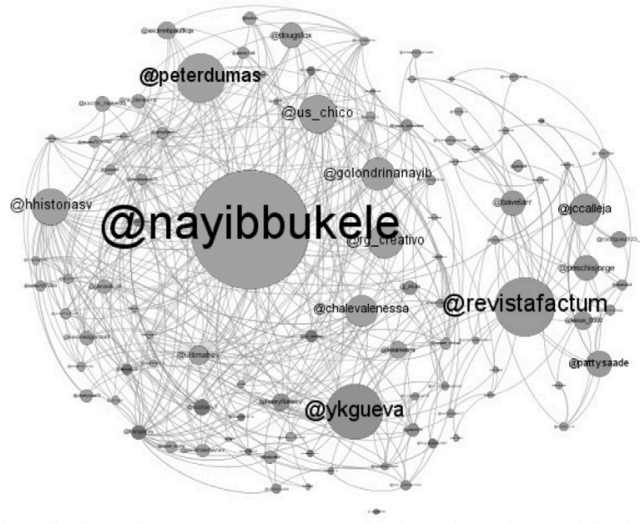

Figura 4. Grafo de comunidades. El grafo presenta las comunidades a la que cada uno de los usuarios pertenece, diferenciados por colores. Las comunidades indican usuarios que comparten características similares dentro de la red, no necesariamente afiliación a un partido político. Fuente: elaboración propia.

\section{Experimento \#3: Día de las elecciones presidenciales}

El día 3 de febrero de 2019 se llevaron a cabo las elecciones presidenciales, por lo que se monitoreó la actividad de cada candidato en sus respectivas cuentas de Twitter desde las 9:25 p. m. a 9:45 p. m. del mismo día; a esa hora ya se habían comunicado los resultados preliminares dictados por el Tribunal Supremo Electoral. De igual manera, se generó la nube de hashtags más utilizados.

\section{Tabla 5}

Parámetros de búsqueda de experimento \#3

\begin{tabular}{|c|c|}
\hline Tipo de búsqueda & Lista de usuarios \\
\hline Términos de búsqueda & $\begin{array}{l}\text { - @jccalleja } \\
\text { - @ nayibbukele } \\
\text { - @ hugomartinezsv } \\
\text { - @josuealvaradosv } \\
\text { - @TSEEISalvador } \\
\text { - @FMLNoficial } \\
\text { - @ARENAOFICIAL } \\
\text { - @GANAOFICIAL } \\
\text { - @ vamoselsalvador }\end{array}$ \\
\hline Tipo de red & Full Twitter Network \\
\hline Tiempo de captura & 20 minutos cada 2 horas \\
\hline
\end{tabular}

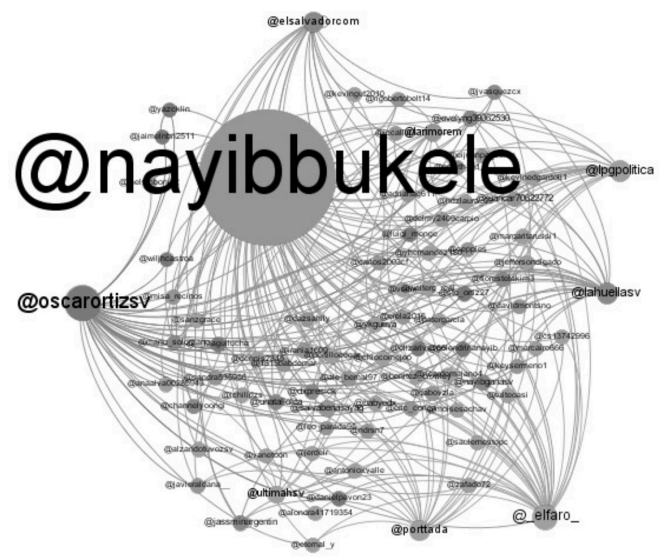

Figura 5. Grafo Nayib Bukele. Este grafo indica 
la actividad e interacción que el candidato tuvo en su cuenta de Twitter de 9:25 p. m. a 9:45 p. m. el día de las elecciones presidenciales. Fuente: elaboración propia.
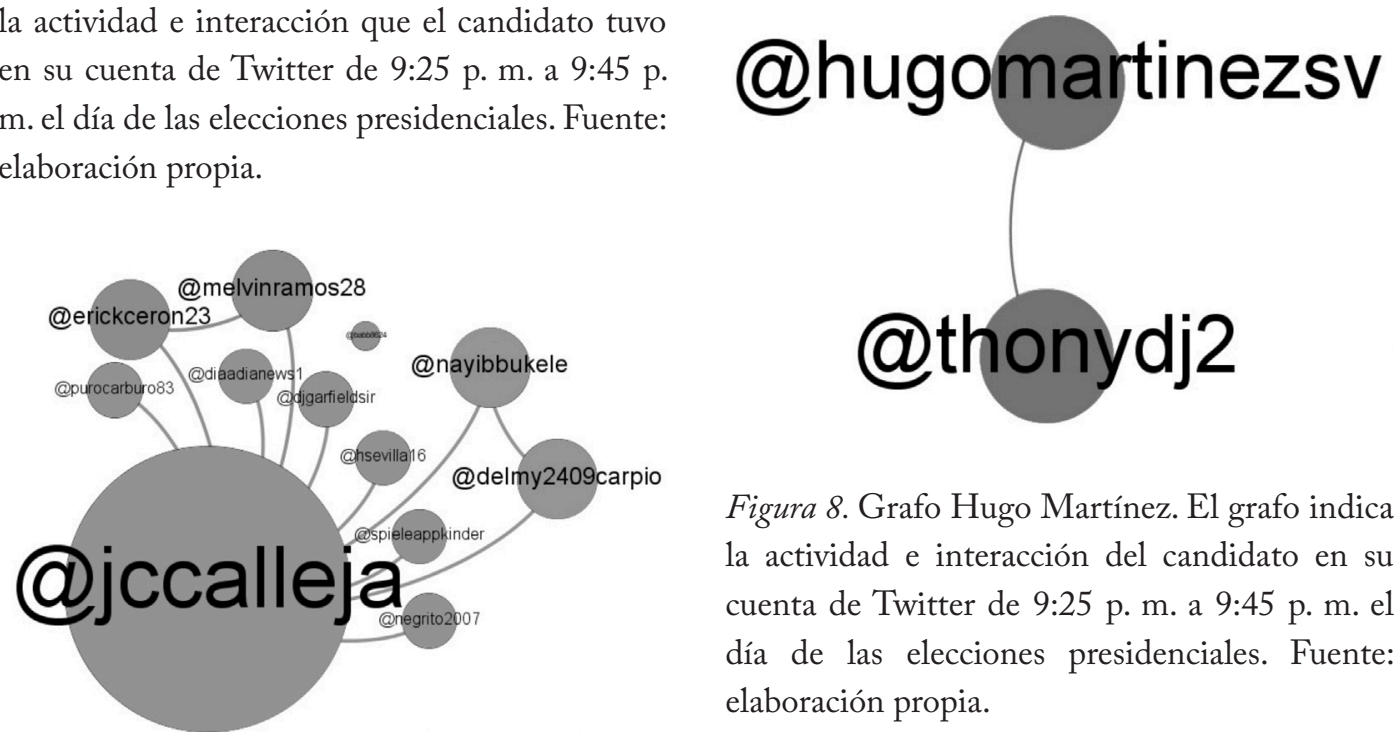

Figura 8. Grafo Hugo Martínez. El grafo indica la actividad e interacción del candidato en su cuenta de Twitter de 9:25 p. m. a 9:45 p. m. el día de las elecciones presidenciales. Fuente: elaboración propia.

Figura 6. Grafo Carlos Calleja. Muestra la actividad e interacción del candidato en su cuenta de Twitter de 9:25 p. m. a 9:45 p. m. el día de las elecciones presidenciales. Fuente: elaboración propia.

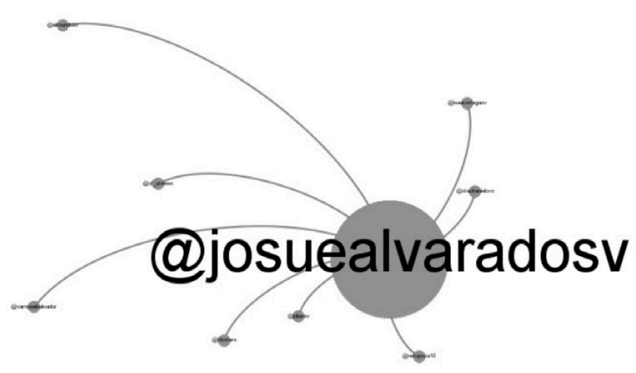

Figura 7. Grafo Josué Alvarado. El grafo representa la actividad e interacción del candidato en su cuenta de Twitter de 9:25 p. m. a 9:45 p. m. día de las elecciones presidenciales. Fuente: elaboración propia.

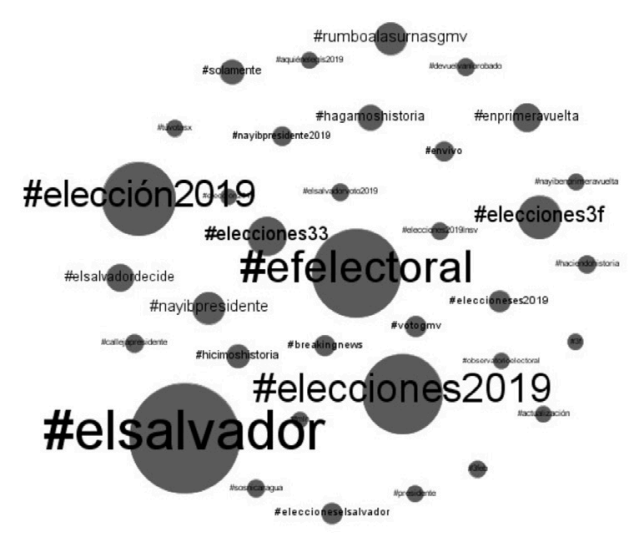

Figura 9. Grafo hashtags. Este grafo muestra los hashtags más utilizados por los usuarios de la red de 9:25 p. m. a 9:45 p. m. el día de las elecciones presidenciales. Fuente: elaboración propia.

\section{Discusión}

Una vez finalizada la captura de datos, Gephi presenta un grafo inicial difícil de visualizar ya que muestra todos los datos recopilados, haciendo complicada su comprensión. Es necesario aplicar las estadísticas y filtros 
mencionados en el método, para obtener un grafo reducido y de esta manera, obtener información valiosa sobre las comunidades que se generan en un evento específico.

Con el experimento \#1 se pudo validar que las medidas estadísticas grado de entrada y grado de salida permiten identificar dentro del grafo los usuarios que tienen un papel de influyente y comunicador, respectivamente. De igual manera, se confirmó que la medida de análisis betweenness identifica los usuarios intermediarios, aquellos que se vuelven el canal para que la información fluya dentro de la red. Un aspecto importante a destacar es que un mismo nodo puede desempeñar varios roles dentro de la red, el nodo identificado como@nayibbukele cumple tanto el rol de intermediario como influyente; por lo tanto, dicho nodo ejecuta los objetivos de mantener distintas comunidades de la red unida, así como ser el nodo con mayor presencia en la red, teniendo la mayor cantidad de retweets, respuestas y/o menciones.

En el experimento \#2 se evidenció la existencia de comunidades virtuales dentro de una misma red de datos. Existe una medida de análisis llamada modularidad, la cual permite partir el grafo en distintas comunidades, las cuales comporten características similares. Dentro del grafo mostrado se visualizan cuatro comunidades, cada una cuenta con un nodo principal, el cual, además de ser el nodo central de una comunidad en especial, es el nodo encargado de crear las conexiones con otras comunidades.

Como se aprecia en el experimento \#3, los grafos generados demostraron que el filtro topología con la opción ego es la herramienta ideal para generar grafos específicos según un parámetro previamente establecido, en este caso, la cuenta de cada uno de los candidatos a la presidencia. Esto permitió poder visualizar la actividad e interacción que un candidato tuvo con sus seguidores en el transcurso del día de las elecciones. Una vez procesado los grafos, se pueden exportar en formato gexf el cual permite crear un sitio web interactivo. Como parte de este experimento invitamos a revisar el recurso web “Twitter el día de las elecciones”. (http:// disruptiva.media/3f/grafo.html).

Los resultados obtenidos en los tres experimentos evidencian la interacción de los usuarios de Twitter. Es importante resaltar que dos usuarios pueden no tener una interacción directa entre ellos y aun así formar parte de la misma red, esto se debe a que el comportamiento de la mayoría de los usuarios consiste en seguir a un actor influyente. De manera que se puede definir una red alrededor del actor influyente y aunque no se conozcan los seguidores, estos mantienen una interacción de manera indirecta.

\section{Conclusiones}

La importancia de estas investigaciones se fundamenta en que este tipo de técnicas de análisis pueden tener múltiples aplicaciones; si bien, para este caso el estudio se centró en el evento mediático de las elecciones presidenciales 2019, este tipo de metodología puede ser implementada por el sector empresarial para conocer el nivel de aceptación que tiene una empresa con sus clientes y a partir de ese análisis, generar sus estrategias de mercado. 
Dando respuesta a la primera interrogante de esta investigación, sobre qué herramientas podemos utilizar para realizar ARS y determinar comunidades virtuales en la plataforma de Twitter. Con los experimentos realizados se logró validar que Gephi es una herramienta con una interfaz muy intuitiva y a la vez efectiva para realizar captura de tweets en tiempo real, tratamiento de los datos y un análisis especializado, permitiendo identificar las comunidades virtuales; así como los distintos roles que cada usuario desempeña dentro la red.

De igual manera, dando respuesta a cuál es el procedimiento para recopilar, analizar y visualizar la información, se llevó a cabo una metodología que inició con la obtención de credenciales de desarrollador en la plataforma de Twitter. Posteriormente se utilizó la herramienta Gephi para recopilar tweets en tiempo real, permitiendo así el monitoreo de eventos mediáticos durante el tiempo en que estos se desarrollaron. Una vez capturados los datos, se aplicaron diversas medidas estadísticas para conocer información relevante de la red, así como distintos filtros que ayudaron a limitar la información únicamente a datos representativos para el análisis.

Como etapa final, se aplicaron algoritmos de distribución para mejorar la visualización, lo que brindó una mejor presentación de los resultados, facilitando la interpretación y comprensión de toda la información procesada.

Finalmente, para futuros trabajos la meta será combinar el análisis de redes sociales con procesamiento de lenguaje natural para analizar el contenido de los tweets y descubrir si el comportamiento de los actores influye positiva o negativamente en el comportamiento de los miembros restantes de la red.

\section{Bibliografía}

Bastian, M., Heymann, S., Jacomy, M. (2009). Gephi: An Open Source Software for Exploring and Manipulating Networks. International AAAI Conference on $W e b$ and Social Media. Obtenido de https://www. aaai.org/ocs/index.php/ICWSM/09/paper/ view/154/1009

Dannbraun. (2017). Nacimiento y evolución de las Redes Sociales. Dannbraun.com. Obtenido de http://www.dannbraun.com/blog/nacimientoevolucion-redes-sociales/

Dettmer, J., Reyna, A. (2014). El análisis de las redes sociales y su aplicación al campo de las Ciencias Sociales. IV Encuentro Latinoamericano de Metodología de las Ciencias Sociales, 27 al 29 de agosto de 2014, Heredia, Costa Rica. Obtenido de http://www.memoria.fahce.unlp.edu.ar/trab_ eventos/ev.8200/ev.8200.pdf

Humberstone Morales, J. (2018). Pescando información en el océano de datos de Twitter. Realidad Y Reflexión(47), 47-57. Obtenido de https://doi.org/10.5377/ryr.v0i47.6273

Knowledgecenter,I.(2018).IBM: Análisis de redes sociales. Obtenido de https://www.ibm.com/ support/knowledgecenter/es/SS3J58_9.1.0/ com.ibm.i2.anb.doc/about_social_network_ analysis.html 
Totet, M. (2016). Twitter Streaming Importer: Naoyun as a Gephi Plugin. Obtenido de matthieu-totet: http://matthieu-totet.fr/ Koumin/2016/04/25/twitter-streamingimporter-naoyun-as-a-gephi-plugin/
Twitter. (2019). Conuming streaming data. Obtenido de https://developer.twitter.com/en/ docs/tutorials/consuming-streaming-data.html

\section{Anexo}

\section{Guía de instalación y configuración de Gephi.}

1. Crear las credenciales de acceso a la API pública de Twitter. Para una guía paso a paso consultar a Humberstone (2018) en el enlace https://doi.org/10.5377/ryr.v0i47.6273

2. Descargar e instalar el programa Gephi 0.92 del sitio web https://gephi.org/users/download/. Consideré que para poder utilizar Gephi es necesario tener instalado Java Runtime Enviroment (JRE) que puede descargar del siguiente enlace https://www.java.com/es/download/.

3. Luego, dentro de Gephi hay que instalar el plugin Twitter Streaming Importer. En la pestaña Herramientas, seleccionar Plugins y dentro de la ventana seleccionar Plugins disponibles. Finalmente, marcar el plugin Twitter Streaming Importer y hacer clic en el botón Instalar.

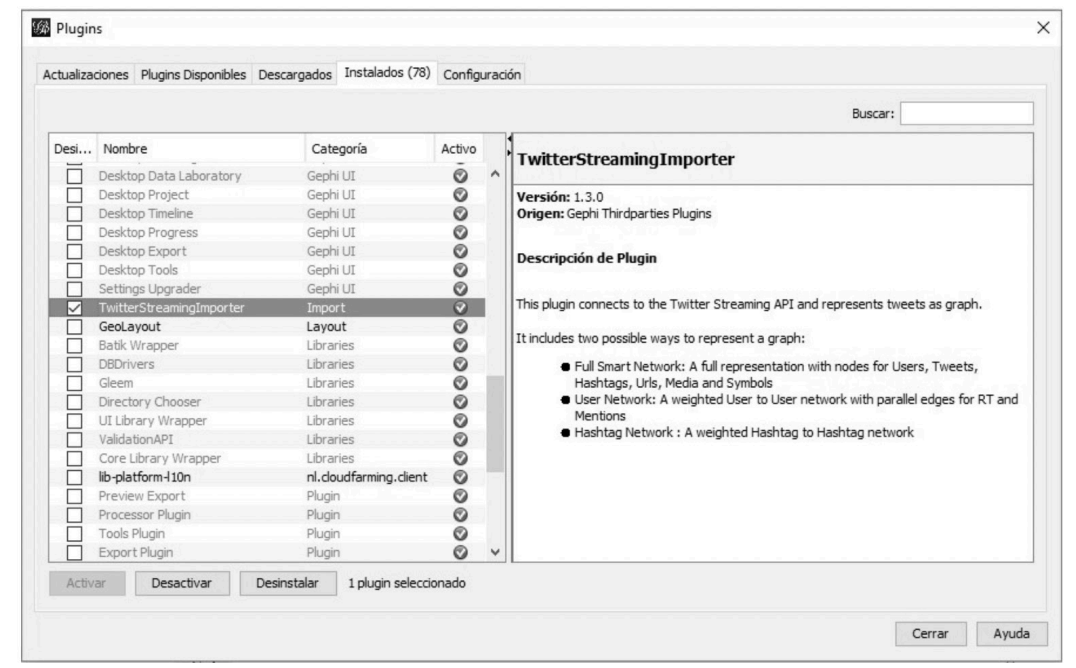

Figura 10. Instalación del plugin Twitter Streaming Importer. Captura de pantalla de la venta de Plugins de la herramienta Gephi. Fuente: elaboración propia. 
4. Una vez descargado el plugin de Twitter, se configuran las credenciales generadas en el primer paso.
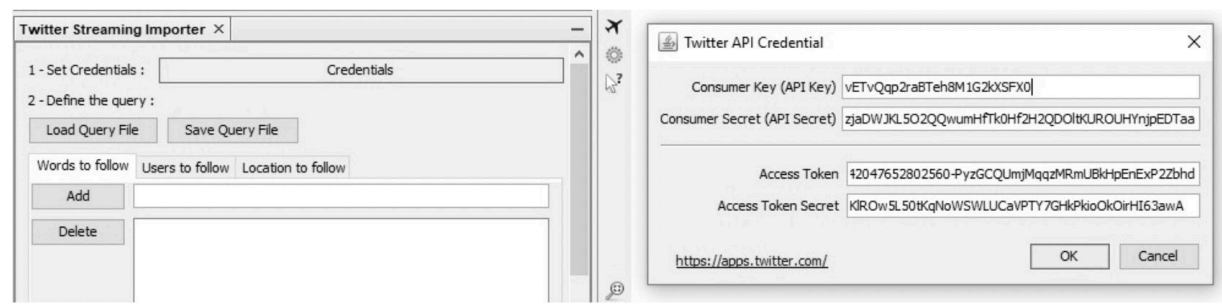

Figura 11. Configuración de credenciales de API Twitter. Captura de pantalla de la venta de configuración de credenciales para utilizar el plugin de Twitter Streaming Importer. Fuente: elaboración propia.

5. Con las credenciales agregadas, se especifican los términos de búsqueda. La opción que se utilizó es Users to follow. Dentro de esta opción se agregó los nombres de los usuarios de Twitter a monitorear en cada experimento. Luego se debe especificar el tipo de captura: solamente de usuarios (User Network) o red completa de Twitter (Full Twitter Network).

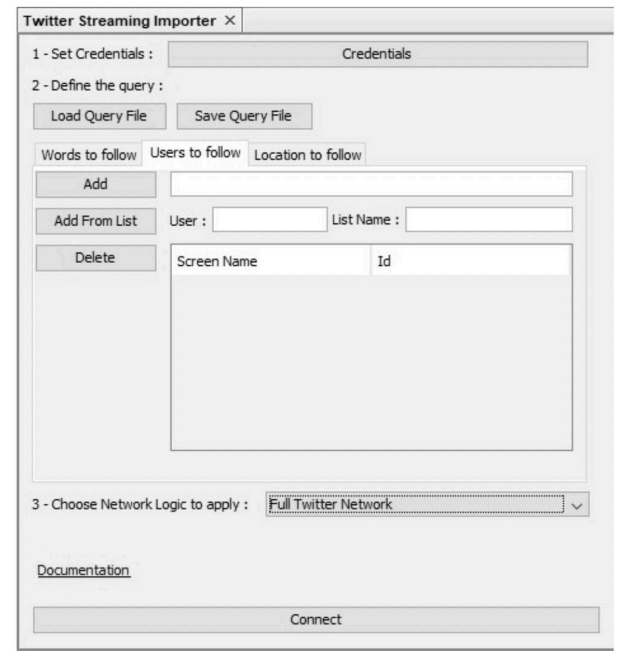

Figura 12. Configuración de parámetros de búsqueda. Captura de pantalla de la venta de configuración de parámetros de búsqueda para utilizar el plugin de Twitter Streaming Importer. Fuente: elaboración propia.

6. Una vez agregado los usuarios y seleccionado el tipo de captura, se procede a conectar a la red de Twitter. La captura puede durar el tiempo que se desee, para detener la captura de datos de la red se selecciona desconectar. 